EPJ Web of Conferences 107, 06004 (2016)

DOI: $10.1051 /$ epjconf/201610706004

(C) Owned by the authors, published by EDP Sciences, 2016

\title{
Charge-exchange resonances and restoration of the Wigner SU(4)-symmetry in heavy and superheavy nuclei
}

\author{
Yu. S. Lutostansky ${ }^{1, a}$, V. N. Tikhonov ${ }^{1}$ \\ ${ }^{1}$ National Research Centre "Kurchatov Institute", 123182, Moscow, Russia
}

\begin{abstract}
Energies of the giant Gamow-Teller and analog resonances $-E_{\mathrm{G}}$ and $E_{\mathrm{A}}$, respectively, - are calculated within the microscopic theory of finite Fermi system. The calculated energy difference $\Delta E_{\mathrm{G}-\mathrm{A}}=E_{\mathrm{G}}-E_{\mathrm{A}}$ tends to zero with $A$ in heavy nuclei indicating the restoration of Wigner SU(4)-symmetry. The calculated $\Delta E_{\mathrm{G}-\mathrm{A}}$ values are in good agreement with the experimental data. The average deviation is $0.30 \mathrm{MeV}$ for the 33 considered nuclei where experimental data are available. The $\Delta E_{\mathrm{G}-\mathrm{A}}$ values are investigated for very heavy and superheavy nuclei up to the mass number $A=290$. Using the experimental data for the analog resonance energies, the isotopic dependence of the Coulomb energy differences for neighboring isobars are analyzed within the SU(4)-approach for more than 400 nuclei in the mass number range of $A=3-244$. The Wigner SU(4)-symmetry restoration for heavy and superheavy nuclei is confirmed. It is shown that the restoration of SU(4)-symmetry does not contradict the possibility of the existence of the "island of stability" in the region of superheavy nuclei.
\end{abstract}

\section{Introduction}

The possible existence of spin-isospin resonance was first discussed in the works by Ikeda, Fujii, and Fujita in the middle of the 1960s as an attempt to explain the observed suppression effect of Gamow-Teller (GT) nuclear transitions [1-3]. They called this state the Gamow-Teller resonance (GTR), in analogy with analog resonance (AR) or Fermi resonance.

The energies and excitation probabilities of these resonances were calculated later for a large group of spherical nuclei [4-6] within the theory of finite Fermi systems (TFFS) [7]. The first results were published in 1972 [4], several years before the experimental observation of the GTR. It was assumed at first that being a spin-flip state, the GTR should be located above the AR by the average energy of spin-orbit splitting $E_{l s}$, and that its width should be the order of $E_{l s}$. Later on it was found from our calculations that the energy splitting between the GTR and $\mathrm{AR}$ is not equal to $E_{l s}$, but it decreases for heavy nuclei. The effect of decreasing the gap between the GTR and AR was first noted in 1973 as a result of calculations for more than 70 isotopes $[5,6]$. We concluded that the Wigner SU(4)-supersymmetry [8] should be restored in heavy nuclei because GTR and AR belongs to the same supermultiplet in this approach, and their main parameters are the same. Since at the time when the GTR was predicted theoretically its energies have not been measured for different nuclei, there were no enough data to verify the hypothesis on Wigner SU(4)-symmetry restoration in heavy nuclei experimentally.

\footnotetext{
a Lutostansky@yandex.ru
}

At present there are three methods to test the hypothesis by comparing the calculated and experimental data on the GTR and AR. The first method is based on the analysis of the degeneration of the Gamow-Teller and analog resonances, as according to SU(4)-approach both the resonances have to belong to the same supermultiplet. The second possibility is related to the realization the FranziniRadicatti relation for nuclear masses [9] following from the SU(4)-theory. The third one is associated with the analysis of the Coulomb energies of nuclei and their isotopic dependence [10]. Analysis of these three possibilities and the restoration of Wigner symmetry was considered recently in [11].

In this paper, we compare the results of our calculations of $\Delta E_{\mathrm{G}-\mathrm{A}}$ values with experimental data and investigate the restoration of Wigner SU(4)-symmetry up to superheavy nuclei with $A=90$. The isotopic dependence of the Coulomb energy difference of neighboring isobars is also analyzed for more than 400 nuclei with available experimental data within the range of mass numbers $A=3-244$. In connection with the restoration of Wigner symmetry in heavy nuclei, the behaviour of the spin-orbit splitting and the associated shell structure become uncertain. These problems are related in its turn with the possibility of the existence of the so-called "island of stability" in the region of superheavy nuclei [12]. This problem is also discussed in the paper.

\section{Method of calculation}

The Gamow-Teller resonance and other charge-exchange excitations in nuclei are described within the TFFS by a 
system of equations for the effective field [7]. For the GT effective nuclear field, we obtain a system of equations that, in the $\lambda$-representation has the form:

$$
\begin{gathered}
V_{\lambda \lambda^{\prime}}=V_{\lambda \lambda^{\prime}}^{\omega}+\sum_{\lambda_{1} \lambda_{2}} \Gamma_{\lambda \lambda^{\prime} \lambda_{1} \lambda_{2}}^{\omega} A_{\lambda_{1} \lambda_{2}} V_{\lambda_{2} \lambda_{1}}+\sum_{v_{1} v_{2}} \Gamma_{\lambda \lambda^{\prime} v_{1} v_{2}}^{\omega} A_{\nu_{1} v_{2}} V_{v_{2} v_{1}} \\
V_{v v^{\prime}}=\sum_{\lambda_{1} \lambda_{2}} \Gamma_{v v^{\prime} \lambda_{1} \lambda_{2}}^{\omega} A_{\lambda_{1} \lambda_{2}} V_{\lambda_{2} \lambda_{1}}+\sum_{v_{1} v_{2}} \Gamma_{v v^{\prime} v_{1} v_{2}}^{\omega} A_{v_{1} v_{2}} V_{v_{2} v_{1}} \\
A_{\lambda \lambda^{\prime}}^{(p \bar{n})}=\frac{n_{\lambda}^{\bar{n}}\left(1-n_{\lambda^{\prime}}^{p}\right)}{\varepsilon_{\lambda}^{\bar{n}}-\varepsilon_{\lambda^{\prime}}^{p}+\omega}, \quad A_{\lambda \lambda^{\prime}}^{(n \bar{p})}=\frac{n_{\lambda}^{\bar{p}}\left(1-n_{\lambda^{\prime}}^{n}\right)}{\varepsilon_{\lambda}^{\bar{p}}-\varepsilon_{\lambda^{\prime}}^{n}-\omega} \\
V_{\mathrm{GT}}^{\omega}=e_{q} \boldsymbol{\sigma \tau} \tau_{+},
\end{gathered}
$$

where $n_{\lambda}$ and $\varepsilon_{\lambda}$ are, respectively, the occupation numbers and energies of $\lambda$-states. The subscripts $v$ are used for the $l$-forbidden part of the interaction. The system of secular equations (1) is obtained from the more general one [13] including the effective changes of pairing gaps $\Delta$ in the $p h$ and $p p$-channels. Here, we use the condition $d_{p n}^{(1)}=d_{p n}^{(2)}=$ 0 assuming that the effects of the pairing gap changes in the external field are negligible, which is justified for the external fields with zero diagonal elements of [7].

Energies of single-particle states and corresponding wave functions are calculated in the shell model for neutrons and protons separately. Pairing correlations are taken into account by replacing $\varepsilon_{\lambda} \rightarrow E_{\lambda}=\sqrt{\varepsilon_{\lambda}^{2}+\Delta_{\lambda}^{2}}$ as in [14]. The energy gaps $\Delta_{\lambda}$ are calculated separately for neutrons and protons.

The quasiparticle effective charge $e_{q}$ is equal to 1 for the Fermi $(\tau \tau)$ transitions and $e_{q}<1$ for the Gamow-Teller $(\sigma \tau)$ transitions. The energies of charge-exchange excitations are defined as the eigenvalues $\omega_{i}$ of secular Eqs. (1).

In the calculations we use a local nucleon-nucleon $\delta$ interaction $\Gamma^{\omega}$ in the Landau-Migdal form with the coupling constants $f_{L}^{\prime}$ and $g_{L}^{\prime}$ of the isospin-isospin $(\tau \tau)$ and the spin-isospin $(\boldsymbol{\sigma} \tau)$ quasiparticle interaction with $L=0$. For the $(\tau \tau)$ coupling constant the value $f_{0}^{\prime}=1.35$ was used determined by the comparison of calculated energy splitting between the analog and anti-analog isobaric states (IS) with the experimental data for a large number of nuclei [15]. For the $(\sigma \tau)$ coupling constant $g_{0}^{\prime}$ we use the value $g_{0}^{\prime}=1.22$ as in the previous calculations [16]. With this value of $g_{0}^{\prime}=1.22$ the experimental differences between the GTR $\left(E_{\mathrm{G} 1}\right)$ and IS $\left(E_{\mathrm{G} 2}\right)$ energies in the Sb isotopes [17] are reproduced. Although the absolute values of the constants can vary in different approaches, the obtained ratio $g_{0}^{\prime} / f_{0}^{\prime}=0.90 \pm 0.03$ is model independent in the TFFS.

The energies of GTR and AR were calculated within the two version of TFFS: a) in the self-consistent TFFS using a simplified version of [18] with the local interaction and $m^{*}=m ; \mathrm{b}$ ) in the approximate version [19] where solutions are obtained in analytical form for collective IS. To this aim we neglect the $l$-forbidden terms in (2) and assume the constancy of the effective field when collective modes excited. If $V(r)=$ const, then for nuclei where $\Delta E=(4 / 3) \varepsilon_{F}(N-Z) / A>E_{l s}^{1}$ or $x=\Delta E / E_{l s}>1$ the

\footnotetext{
${ }^{1}$ The Fermi energy $\varepsilon_{F} \approx 40 \mathrm{MeV}$
}

energy difference $\Delta E_{\mathrm{G}-\mathrm{A}}$, normalized to the spin-orbital splitting $E_{l s}$, is the following:

$$
\begin{aligned}
& y=\frac{\Delta E_{\mathrm{G}-\mathrm{A}}}{E_{l s}} \approx\left(g_{0}^{\prime}-f_{0}^{\prime}\right) x+b \frac{1+b g_{0}^{\prime}}{g_{0}^{\prime} x\left(1+c_{\mathrm{A}} / x^{2}\right)}, \\
& E_{l s}=\sum_{\lambda_{1} \lambda_{2}} n_{\lambda_{1}}\left(1-n_{\lambda_{2}}\right) \varepsilon_{\lambda_{1} \lambda_{2}}^{l s} / \sum_{\lambda_{1} \lambda_{2}} n_{\lambda_{1}}\left(1-n_{\lambda_{2}}\right),
\end{aligned}
$$

and

$$
b=\frac{2}{3}\left[1-(2 A)^{-1 / 3}\right], \quad c_{A}=0.8 A^{-1 / 3} .
$$

In Eq. (2), the average value of spin-orbit splitting $E_{l s}$ is a parameter that can be calculated either from the singleparticle scheme of GT spin-flip transitions, as was done in $[6,18]$, or can be obtained phenomenologically by comparing calculated $\Delta E_{\mathrm{G}-\mathrm{A}}$ with the experimental data [16]. For the dependence of $E_{l s}$ on the neutron number $N$ in heavy nuclei with $N>80$ the following parametrization is used:

$$
E_{l s}=20 N^{-1 / 3}+1.25 \mathrm{MeV} .
$$

Equation (3) is obtained as in [20] but substituting $A$ for $N$. It corresponds to decreasing of the $E_{l s}$ value and restoration of the Wigner SU(4)-symmetry in heavy nuclei.

Equation (2) is also applicable for very heavy and superheavy nuclei, because for them the value of $x=\Delta E / E_{l s}$ is larger and the accuracy of the calculations should be better.

\section{Results and discussion}

Energy differences between the Gamow-Teller and analog resonances $\Delta E_{\mathrm{G}-\mathrm{A}}$ were calculated with Eq. (2) for 33 nuclei: ${ }^{48} \mathrm{Ca},{ }^{60,64} \mathrm{Ni},{ }^{71} \mathrm{Ga},{ }^{76} \mathrm{Ge},{ }^{82} \mathrm{Se},{ }^{90,91,92,94} \mathrm{Zr},{ }^{93} \mathrm{Nb}$, $94,96,97,98,100 \mathrm{Mo}, \quad{ }^{115} \mathrm{In}, \quad{ }_{112,114,116,117,118,119,120,122,124} \mathrm{Sn}$, ${ }^{128,130} \mathrm{Te},{ }^{127} \mathrm{I},{ }^{136} \mathrm{Xe},{ }^{150} \mathrm{Nd},{ }^{169} \mathrm{Tm}$ and ${ }^{208} \mathrm{~Pb}$ (the target nuclei), for which experimental data are available [16, 19]. Calculated and experimental values of $y(x)=\Delta E_{\mathrm{G}-\mathrm{A}} / E_{l s}$ as a function of the dimensionless parameter $x$ defined above are presented in Fig. 1. The leftmost and rightmost points correspond to ${ }^{60} \mathrm{Ni}$ and ${ }^{208} \mathrm{~Pb}$ with $x=0.52$ and $x=2.15$, respectively. The differences between the calculated and experimental values of $\Delta E_{\mathrm{G}-\mathrm{A}} \Delta \varepsilon=\left|\Delta E_{\mathrm{G}-\mathrm{A}}^{\text {th }}-\Delta E_{\mathrm{G}-\mathrm{A}}^{\text {exp }}\right|$ is $0.38 \mathrm{MeV}$ for ${ }^{60} \mathrm{Ni}$ and $\lesssim 0.10 \mathrm{MeV}$ for ${ }^{208} \mathrm{~Pb}$, indicating that the accuracy of calculations improves for heavy nuclei. The r.m.s. deviation of the theoretical results obtained by Eq. (2) from the experimental data is $\delta(\Delta \varepsilon) \leq 0.30 \mathrm{MeV}$ which is comparable to the accuracy of the $E_{\mathrm{GTR}}$ experimental data [17].

Figure 1 also shows calculations for nuclei located at the "line of beta-stability" which is determined by the formula

$$
Z_{\beta}=A /\left(2+0.015 A^{2 / 3}\right)
$$

Equation (4) was derived in [21] from the condition $\partial M / \partial Z=0$ (at constant $A$ ), using well-known formula for the nuclear binding energy in the liquid-drop model. Here, $Z_{\beta}$ corresponds to the nucleus with the smallest mass within each isobaric chain. 


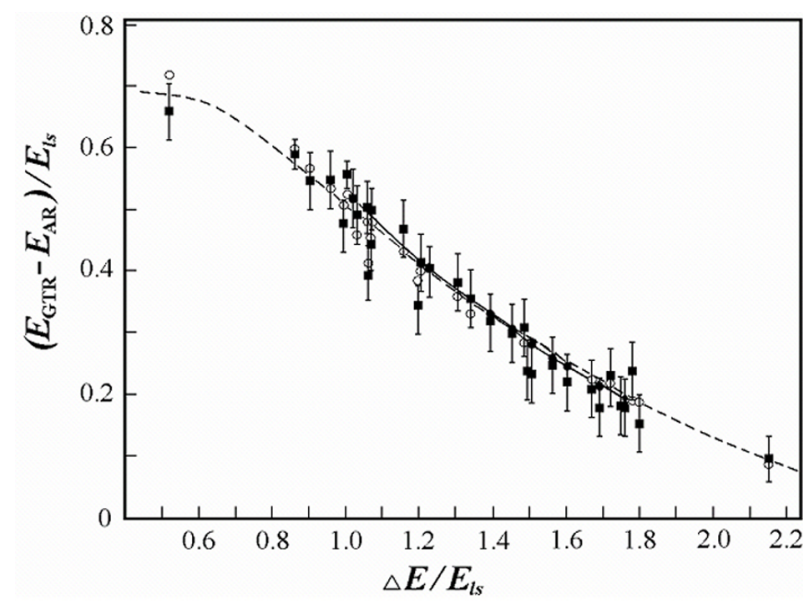

Figure 1. Dependence of the normalized energy difference between the GTR and AR $y(x)=\left(E_{\mathrm{GTR}}-E_{\mathrm{AR}}\right) / E_{l s}$ on the value $x=\Delta E / E_{l s}$ calculated by Eq. (2) (open circles) and the corresponding experimental data (black squares). Black dots connected by the line represent the values calculated for $\mathrm{Sn}$ isotopes. The dashed line shows the $y(x)$ values calculated with the $E_{l s}$ obtained by Eq. (3) for nuclei located on the beta-stability line determined by Eq. (4).

\section{Analog resonances and Coulomb displacement energies in SU(4)-approach}

If the analog and Gamow-Teller resonances belong to the same supermultiplet, the AR energies should be described in the framework of SU(4)-theory. The corresponding analysis was performed in [10], where the possibility to describe the difference $\Delta E_{C}$ between the Coulomb energies of neighboring isobar nuclei within the SU(4)-theory was demonstrated. However, in Ref. [10] only the nuclei with $A<60$ were considered and an unambiguous conclusion was not made. We analyzed the $\Delta E_{C}$ values for more than 400 nuclei in the mass number interval $A=3$ 244 for which experimental data are known (see [22]). As in $[10,22]$, we used the two-parameter formula

$$
\Delta E_{\mathrm{C}}=E_{\mathrm{C}}(A, Z+1)-E_{\mathrm{C}}(A, Z)=a \frac{Z}{A^{1 / 3}}+b .
$$

We obtain $a=1416$ and $b=-698$ (both in $\mathrm{keV}$ ) with the standard deviation $\delta E<100 \mathrm{keV}$. Deformation was taken into account phenomenologically as in [22], by introducing the correction to $\Delta E_{C}^{d e f}=\Delta E_{C}^{s p h}-\delta E_{C}^{d e f}$ with the deformation parameters $\beta_{2}$ and $\beta_{4}$ from [23]. In the SU(4)scheme, four types of ground states of nuclei belong to different supermultiplets: (i) ( $Z$-even, $N$-even) nuclei belong to the $\left(T_{Z}, 0,0\right)$ supermultiplet; (ii) (Z-even, $N$-odd) nuclei belong to the $\left(T_{Z}, 1 / 2,1 / 2\right)$ supermultiplet; (iii) ( $Z$ odd, N-even) nuclei belong to the $\left(T_{Z}, 1 / 2,-1 / 2\right)$ supermultiplet; and (iv) (Z-odd, $N$-odd) nuclei belong to the $\left(T_{Z}\right.$, $1,0)$ supermultiplet where the isospin $T_{Z}=(N-Z) / 2$. The energy $\Delta E_{\mathrm{C}}$ is considered as the difference between the energy of the ground state of the $(A, Z)$ nucleus and the energy of excitation of the analog resonance in the
$(A, Z+1)$ nucleus taking into account the energy of $\beta$ decay $Q_{\beta}$. Correspondingly, taking into account the mass difference $\Delta M=M_{n}-M_{\mathrm{H}}=782.35 \mathrm{keV}$, we obtain the relation $b=\beta-\Delta M$ for the parameter $b$ from Eq. (5), where the parameter $\beta$ in the SU(4)-scheme should depend on the supermultiplet of the ground state. In particular, for nuclei with even $Z$, i.e., for cases (i) and (ii), the equality $\beta=0$ should be satisfied [10]. This equality is really observed: the average deviation of the $\beta$ value from zero is $80 \mathrm{keV}$. The most interesting cases are $Z$-odd nuclei for which the $\mathrm{SU}(4)$-scheme gives the dependence $\beta=\alpha / T_{Z}$ on the isospin $T_{Z}$, where the parameter $\alpha$ is different for $N$-even and $N$-odd nuclei [10]. The analysis shows the inverse dependence $\beta \approx 83 / T_{Z} \mathrm{keV}$ for nuclei with odd $Z$ values according to the SU(4)-approach [10]. However, we were not able to obtain different $\alpha$ values for different supermultiplets because of insufficient data on $\Delta E_{\mathrm{C}}$ for odd-odd nuclei.

Nevertheless, analyzing the experimental data on the energies $\Delta E_{\mathrm{C}}$ for more than 400 nuclei, one can state that the observed functional dependence corresponds to the SU(4)-theory.

\section{Energies of Gamow-Teller and analog resonances in heavy and superheavy nuclei}

Equations (2) and (3) are also valid for heavy and superheavy $(\mathrm{SH})$ nuclei and provide even better results because the parameter $x=\Delta E / E_{l s}$ is larger in this case and the conditions of the approach applicability (see Eq. (2) and related notes) are better.

Figure 2 shows the results of the calculations of the absolute value $\Delta E_{\mathrm{G}-\mathrm{A}}$ as a function of the mass number for nuclides with $A>140$ located on the line of beta stability. These nuclides with $Z_{\beta}(A)$ were determined for each isobaric chain as the nucleus with the minimum mass from the experimental data [24]. The microscopic calculations of the energy differences between the Gamow-Teller and analog resonances for the ${ }^{257} \mathrm{Fm},{ }^{271} \mathrm{Sg},{ }^{280} \mathrm{Ds}$, and ${ }^{290} \mathrm{Lv}$ nuclei with allowance for the single-particle structure, as in [25], are also presented. According to Eq. (2), these calculations are approximate because the deformation of nuclei was taken into account phenomenologically, as in [22]. Meanwhile, the consistent inclusion of deformation should affect the single-particle spectrum. However, the effect of deformation on the value of spin-orbit splitting, which determines the position of the Gamow-Teller resonance, is small. It was found that the energies $\Delta E_{\mathrm{G}-\mathrm{A}}$ calculated by Eq. (1) for four heavy nuclei differ within $0.1 \mathrm{MeV}$ from those calculated by Eq. (2). As seen in Fig. 2, the Gamow-Teller and analog resonances are also degenerate for heavy nuclei. However, the $\Delta E_{\mathrm{G}-\mathrm{A}}$ values microscopically calculated according to Eq. (2) are somewhat larger than those obtained by Eq. (2) for nuclei on the line of beta stability because of a more correct calculation of the energy $E_{l s}$ from the single-particle level scheme. The analysis of spin-orbit splitting in superheavy nuclei performed in [26] within the generalized self-consistent 


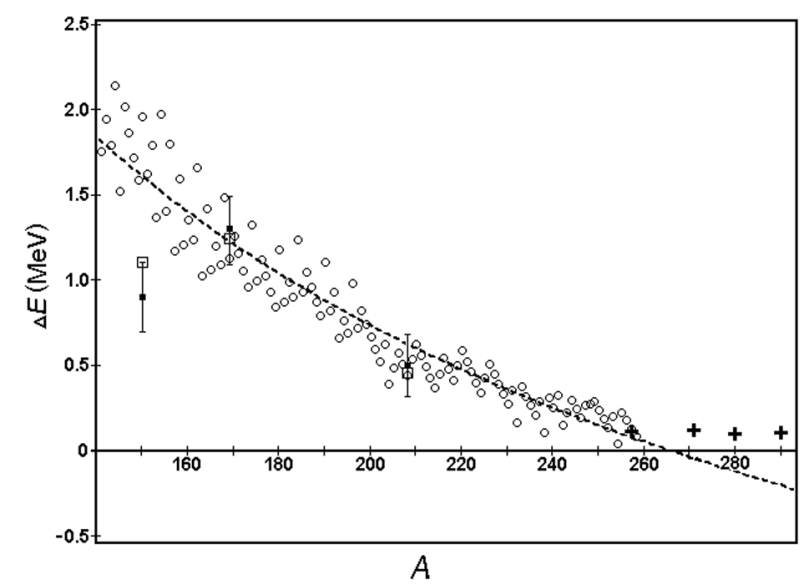

Figure 2. Energy difference $\Delta E_{\mathrm{G}-\mathrm{A}}$ versus mass number A. 1) Theoretical calculations by Eq.(2) (open squares) and experimental values for the ${ }^{150} \mathrm{Nd},{ }^{169} \mathrm{Tm}$, and ${ }^{208} \mathrm{P}$ nuclei (black squares with error bars). 2) $\Delta E_{\mathrm{G}-\mathrm{A}}$ calculated for nuclei located on the line of beta stability from [24] (circles). 3) $\Delta E_{\mathrm{G}-\mathrm{A}}$ calculated for nuclei on the line of beta stability determined by Eq. (4) (the dashed line). 4) $\Delta E_{\mathrm{G}-\mathrm{A}}$ calculated for the ${ }^{257} \mathrm{Fm},{ }^{271} \mathrm{Sg}$, ${ }^{280} \mathrm{Ds}$, and ${ }^{290} \mathrm{Lv}$ nuclei by Eq. (2) (crosses).

method of the energy density functional showed that the consistent variation of the spin-orbit interaction parameters slightly affects the spin-orbit splitting; i.e., this quantity is rather stable. Some decreasing of spin-orbit splitting in very neutron-rich nuclei near the drip-line was predicted earlier [27], but this did not destroy their shell structure.

Thus, taking into account a degeneracy in the matrix elements of the Gamow-Teller and analog resonances [28], one can conclude that a decrease in the energy difference $\Delta E_{\mathrm{G}-\mathrm{A}}$ between the Gamow-Teller and analog resonances in heavy nuclei is due to the restoration of SU(4)symmetry and both resonances belong to the same Wigner supermultiplet together with the ground state of the parent $(A, Z)$ nucleus.

\section{Conclusions}

In the present paper we investigated the difference of the Gamow-Teller and analog resonance energies $\Delta E_{\mathrm{G}-\mathrm{A}}$ and its dependence on the nuclear mass number $A$. The calculations were made in the framework of different apoximations of TFFS. A good agreement with experimental data was found. For 33 considered nuclei with known experimental data the root-mean-square deviation of theoretical and experimental results is $\sim 0.30 \mathrm{MeV}$. The convergence of GTR and AR energies for the group of heavy nuclei with $Z \geq 100$ on the beta-stability line was shown. On the base of observed degeneration of Gamow-Teller and ana$\log$ resonances in heavy nuclei and prediction of this effect for superheavies, we conclude that the Wigner SU(4)restoration is confirmed. This allows to describe the heavy nuclei properties more confidently using SU(4) theory, especially for mass relations [29].
Our conclusions are confirmed by the mass relations and Coulomb energies. The analysis of the FranziniRadikatti relation [9] for nuclear masses, resulting from the SU(4)-theory, was performed several times [30, 31] and shows that these relations works better in heavier nuclei. Also the analysis of the Coulomb displacement energies within the SU(4)-approach allows one to describe the mass and energies of superheavy nuclei with good accuracy.

Thus, it is shown that Wigner SU(4) symmetry is restored in heavy nuclei. As a result, the interpretation of the spin-orbit splitting and the corresponding shell structure, as well as the possibility of existence of the "island of stability" in the region of superheavy nuclei, become indefinite. Our analysis of the degeneracy of the Gamow-Teller and analog resonances involves the ratio $x=\Delta E / E_{l s}$, which grows in heavy nuclei with the energy $\Delta E \sim(N-Z) / A$ even at the constant value of $E_{l s}$. Here, $E_{l s}$ is the average energy of spin-flip single-particle transitions within spin-orbit doublets (3), which decreases with an increase of the neutron excess. According to estimate (3), the energy $E_{l s}$ tends to a finite value in heavy nuclei and does not vanish. The microscopic calculations for superheavy nuclei (see Fig. 2) confirm that $E_{l s}$ is greater than zero and even increases slightly when approaching the "island of stability". In the previous calculations [32] in the self-consistent theory was obtained shell stability for superheavy increased for spherical nuclei with $N \approx 184$ and $Z>114$.

We may conclude that the restoration of Wigner SU(4) symmetry in heavy nuclei does not contradict the possibility of the existence of the "island of stability" in the region of superheavy nuclei [33].

\section{Acknowledgements}

The authors are grateful to S.S. Gershtein, E.E. Sapershtein, N.B. Shulgina, S.V. Tolokonnikov and D.M. Vladimirov for their assistance and helpful discussions.

This work was partly supported by the Russian Foundation for Basic Research, project no's. 13-02-12106 ofi-m (Section 5), 14-22-03040 ofi-m (Section 4) and Swiss National Science Foundation grant no IZ73Z0_152485 SCOPES (Section 1).

\section{References}

[1] K. Ikeda, S. Fujii, and J.I. Fujita, Phys. Lett. 2, 169 (1962); 3, 271 (1963)

[2] J.I. Fujita, S. Fujii, and K. Ikeda, Phys. Rev. B 133, 549 (1964)

[3] J.I. Fujita and K. Ikeda, Nucl. Phys. A 67, 145 (1965)

[4] Yu.V. Gaponov and Yu.S. Lyutostanskii, JETP Lett. 15, 120 (1972)

[5] Yu.V. Gaponov and Yu.S. Lyutostanskii, JETP Lett. 18, 75 (1973)

[6] Yu.V. Gaponov and Yu.S. Lyutostanskii, Sov. J. Nucl. Phys. 19, 33 (1974)

[7] A.B. Migdal, Theory of Finite Fermi Systems and Applications to Atomic Nuclei (Nauka, Moscow, 1983; Interscience, New York, 1967). 
[8] E.P. Wigner, Phys. Rev. 51, 106 (1937); 56, 519 (1939)

[9] P. Franzini and L. A. Radicatti, Phys. Lett. 6, 322 (1963)

[10] D.M. Vladimirov and N.B. Shulgina, Sov. J. Nucl. Phys. 45, 983 (1987)

[11] Yu.S. Lutostansky, V.N. Tikhonov, JETP Lett. 102, 7 (2015)

[12] Yu.Ts. Oganessian, Radiation Physics and Chemistry, 61, 259 (2001)

[13] I. N. Borzov, S. A. Fayans, E. L. Trykov, Nucl. Phys. A 584, 335 (1995)

[14] Yu.S. Lutostansky, N.B. Shulgina, Phys. Rev. Lett. 67, 430 (1991)

[15] Yu.V. Gaponov and Yu.S. Lyutostanskii, Sov. J. Nucl. Phys. 16, 207 (1972)

[16] Yu.S. Lutostansky and V.N. Tikhonov, Bull. Russ. Acad. Sci. Phys., 78, 373 (2014)

[17] K. Pham, J. Jdnecke, D.A. Roberts, et al., Phys. Rev. C 51, 526 (1995)

[18] Yu.V. Gaponov and Yu.S. Lyutostanskii, Phys. Part. Nucl. 12, 528 (1981)

[19] Yu.S. Lutostansky, Phys. At. Nucl. 74, 1176 (2011)

[20] K. Nakayama, A. Pio Galeao, and F. Krmpotic, Phys. Lett. B 114, 217 (1982)
[21] Yu.S. Lyutostanskii, Izv. Akad. Nauk SSSR. Ser. Fiz. 50, 834 (1986)

[22] Yu.S. Lutostansky and V.N. Tikhonov, Bull. Russ. Acad. Sci. Phys. 79, 425 (2015)

[23] P. Möller, J.R. Nix, W.D. Myers, W.J. Swiatecki, At. Data \& Nucl. Data Tables 59, 185 (1995)

[24] M. Wang, G. Audi, A.H. Wapstra, et al., Chinese Phys. C 36, 1603 (2012)

[25] S.V. Tolokonnikov, Yu.S. Lutostansky, and E.E. Saperstein, Phys. At. Nucl. 76, 708 (2013)

[26] S.V. Tolokonnikov and E.E. Saperstein, Phys. At. Nucl. 73, 1684 (2010)

[27] J. Dobaczewski, I. Hamamoto, W. Nazarewicz, and J.A. Sheikh, Phys. Rev. Lett. 72, 981 (1994)

[28] Yu.V. Gaponov, JETP Lett. 38, 240 (1983)

[29] Yu.V. Gaponov, N.B. Shulgina, D.M. Vladimirov, Nucl. Phys. A 391, 93 (1982)

[30] Yu.V. Gaponov, Yu. I. Grigor'yan, Yu. S. Lyutostansky, Sov. J. Nucl. Phys. 31, 34 (1980)

[31] A.M. Nurmukhamedov, Phys. At. Nucl. 75, 29 (2012)

[32] J. Dobaczewski et al., Nucl. Phys. A 611, 211 (1996)

[33] Yu.Ts. Oganessian and V.K. Utyonkov, Rep. Prog. Phys. 78, 036301 (2015) 